\title{
Small deviations of a Galton-Watson process with immigration
}

\author{
NADIA SIDOROVA \\ Department of Mathematics, University College London, Gower Street, London WC1 E6BT, UK. \\ E-mail: n.sidorova@ucl.ac.uk
}

\begin{abstract}
We consider a Galton-Watson process with immigration $\left(\mathcal{Z}_{n}\right)$, with offspring probabilities $\left(p_{i}\right)$ and immigration probabilities $\left(q_{i}\right)$. In the case when $p_{0}=0, p_{1} \neq 0, q_{0}=0$ (that is, when $\operatorname{essinf}\left(\mathcal{Z}_{n}\right)$ grows linearly in $n$ ), we establish the asymptotics of the left tail $\mathbb{P}\{\mathcal{W}<\varepsilon\}$, as $\varepsilon \downarrow 0$, of the martingale limit $\mathcal{W}$ of the process $\left(\mathcal{Z}_{n}\right)$. Further, we consider the first generation $\mathcal{K}$ such that $\mathcal{Z}_{\mathcal{K}}>\operatorname{essinf}\left(\mathcal{Z}_{\mathcal{K}}\right)$ and study the asymptotic behaviour of $\mathcal{K}$ conditionally on $\{\mathcal{W}<\varepsilon\}$, as $\varepsilon \downarrow 0$. We find the growth scale and the fluctuations of $\mathcal{K}$ and compare the results with those for standard Galton-Watson processes.
\end{abstract}

Keywords: conditioning; Galton-Watson processes; Galton-Watson trees; immigration; large deviations; lower tail; martingale limit; small value probabilities

\section{Introduction and main results}

\subsection{Galton-Watson process without immigration}

Let $\left(Z_{n}: n \geq 0\right)$ be a supercritical Galton-Watson process with a non-degenerate offspring random variable $X$. We denote the offspring probabilities by $\left(p_{k}: k \geq 0\right)$ and assume that $p_{0}=0$. Further, we assume that the process starts with one ancestor, that is, $Z_{0}=1$, and denote by $a=\mathbb{E} X>1$ the average offspring number.

It is well known by the Kesten-Stigum theorem that under the condition $\mathbb{E} X \log X<\infty$ the martingale limit

$$
W=\lim _{n \rightarrow \infty} \frac{Z_{n}}{a^{n}}
$$

exists and is strictly positive almost surely. Moreover, the random variable $W$ has a strictly positive continuous density, see [2]. However, only in a very limited number of examples can the distribution of $W$ be computed explicitly, and one has to rely on asymptotic results to describe the behaviour of $W$.

The left tail asymptotics $\mathbb{P}\{W<\varepsilon\}$ as $\varepsilon \downarrow 0$ of $W$ has attracted a lot of mathematical attention, both in its own right and in the broader context of small value problems, see [12]. Since small values of the martingale limit $W$ correspond to sub-average branching of the Galton-Watson tree, one naturally has to distinguish between the Schröder case when $p_{1}>0$ and the Böttcher case when $p_{1}=0$. In the Schröder case, small values of $Z_{n}$ are achieved much more easily and, in particular, the minimal value of $Z_{n}$ is equal to one with positive probability. In sharp contrast to that, in the Böttcher case the minimal tree grows exponentially. 
For two real-valued functions $h_{1}$ and $h_{2}$ defined on $\left(0, \varepsilon_{0}\right)$ for some $\varepsilon_{0}>0$ and such that $h_{2}(\varepsilon) \neq 0$ for all $\varepsilon$ small enough, we say that $h_{1} \sim h_{2}$ as $\varepsilon \downarrow 0$ if $\lim _{\varepsilon \downarrow 0} \frac{h_{1}(\varepsilon)}{h_{2}(\varepsilon)}=1$ and we say that $h_{1} \asymp h_{2}$ as $\varepsilon \downarrow 0$ if there are $c, \hat{c}>0$ such that $c<\frac{h_{1}(\varepsilon)}{h_{2}(\varepsilon)}<\hat{c}$ for all sufficiently small $\varepsilon$.

The Schröder case was first studied in [8], where it was shown that

$$
\mathbb{E} e^{-s W} \asymp s^{-\tau} \quad \text { as } s \rightarrow \infty,
$$

with $\tau=-\frac{\log p_{1}}{\log a}$. Shortly after that in [9] it was proved that the density $w$ of $W$ decays at zero as $w(\varepsilon) \asymp \varepsilon^{\tau-1}$, which easily implies

$$
\mathbb{P}\{W<\varepsilon\} \asymp \varepsilon^{\tau} \quad \text { as } \varepsilon \downarrow 0 .
$$

The asymptotics for the density $w$ was then refined in [5] to $w(\varepsilon) \sim \hat{L}(\varepsilon) \varepsilon^{\tau-1}$ with an analytic multiplicatively periodic function $\hat{L}$, which led to the corresponding improvement of the left tail asymptotics

$$
\mathbb{P}\{W<\varepsilon\} \sim L(\varepsilon) \varepsilon^{\tau} \quad \text { as } \varepsilon \downarrow 0
$$

with another analytic multiplicatively periodic function $L$.

In the Böttcher case, the situation is more complicated as the tail of $W$ decays exponentially. It was shown in [8] that the Laplace transform of $W$ at infinity has the logarithmic asymptotics

$$
\log \mathbb{E} e^{-s W} \asymp s^{\beta} \quad \text { as } s \rightarrow \infty,
$$

where $\beta=\frac{\log \mu}{\log a}$ with $\mu=\min \left\{n: p_{n}>0\right\} \geq 2$ being the minimal offspring number. This suggested the logarithmic asymptotics

$$
\log \mathbb{P}\{W<\varepsilon\} \asymp \varepsilon^{-\frac{\beta}{1-\beta}} \quad \text { as } \varepsilon \downarrow 0
$$

and a more precise result was then obtained in [5], namely, that

$$
\log \mathbb{P}\{W<\varepsilon\} \sim-M(\varepsilon) \varepsilon^{-\frac{\beta}{1-\beta}} \quad \text { as } \varepsilon \downarrow 0,
$$

where $M$ is an analytic positive multiplicatively periodic function. A numerical example with tiny but non-trivial oscillations in $M$ was provided in [3], and an example with a constant $M$ was given in [11]. Finally, the full left tail asymptotics was computed in [10] to be

$$
\mathbb{P}\{W<\varepsilon\} \sim \hat{M}(\varepsilon) \varepsilon^{\frac{\beta}{2(1-\beta)}} \exp \left\{-M(\varepsilon) \varepsilon^{-\frac{\beta}{1-\beta}}\right\} \quad \text { as } \varepsilon \downarrow 0,
$$

where both $M$ and $\hat{M}$ are analytic, positive, and multiplicatively periodic.

The precise form of the above asymptotics as well as the approach developed in [10] made it possible to understand the influence of small values of $W$ on the Galton-Watson tree. Let

$$
K=\min \left\{n: Z_{n}>\mu^{n}\right\}
$$


be the first generation, where a vertex has more than the minimal number of offspring. We will call this event the first non-trivial branching of the tree. It was shown in [4] that, conditionally on $W<\varepsilon$, the first branching time $K$ will grow in the Schröder case as

$$
\gamma_{\mathrm{s}}(\varepsilon)=\frac{\log (1 / \varepsilon)}{\log a}
$$

and in the Böttcher case as

$$
\gamma_{\mathrm{b}}(\varepsilon)=\frac{\log (1 / \varepsilon)}{\log (a / \mu)}-\frac{\log \log (1 / \varepsilon)}{\log a}+H(\varepsilon)
$$

for some continuous multiplicatively periodic function $H$. However, more striking are the fluctuations of $K$. It was proved in [4] that in the Böttcher case there are no fluctuations at all, with $K$ being equal to either $\left\lfloor\gamma_{\mathrm{b}}(\varepsilon)\right\rfloor$ or $\left\lfloor\gamma_{\mathrm{b}}(\varepsilon)\right\rfloor+1$ with probability tending to 1 , conditionally on $W<\varepsilon$. This is no longer true for the Schröder case. Our first result below shows that the random variable $K-\gamma_{\mathrm{s}}(\varepsilon)$ conditioned on $W<\varepsilon$ has exponentially decaying left and right tails.

Theorem 1.1. In the Schröder case, as $x \rightarrow \infty$,

$$
\liminf _{\varepsilon \downarrow 0} \mathbb{P}\left\{K-\gamma_{\mathrm{s}}(\varepsilon)>x \mid W<\varepsilon\right\} \asymp \underset{\varepsilon \downarrow 0}{\limsup } \mathbb{P}\left\{K-\gamma_{\mathrm{s}}(\varepsilon)>x \mid W<\varepsilon\right\} \asymp p_{1}^{x}
$$

and

$$
\liminf _{\varepsilon \downarrow 0} \mathbb{P}\left\{K-\gamma_{\mathrm{s}}(\varepsilon)<-x \mid W<\varepsilon\right\} \asymp \limsup _{\varepsilon \downarrow 0} \mathbb{P}\left\{K-\gamma_{\mathrm{s}}(\varepsilon)<-x \mid W<\varepsilon\right\} \asymp p_{1}^{(\lambda-1) x},
$$

where $\lambda=\min \left\{k>1: p_{k} \neq 0\right\}$.

Remark 1. It was shown in [4] that both tails of $K-\gamma_{\mathrm{s}}(\varepsilon)$ conditioned on $W<\varepsilon$ are not heavier than exponential but it was not known whether this estimate is sharp for either of the tails, and if so what the correspondent exponents and rates are. To find the actual tail behaviour, we had to control the error term of the asymptotics (1.1).

Remark 2. Under the conditioning $W<\varepsilon$ there are two competing effects influencing the behaviour of $K$ : branching too early would lead to higher values of $W$ but on the other hand it would be probabilistically expensive to suppress branching for too long. Having first branching in generation roughly equal to $\gamma_{\mathrm{s}}(\varepsilon)$ corresponds to the optimal trade-off between these two effects. The right tail of $K-\gamma_{\mathrm{s}}(\varepsilon)$ corresponds to a late branching, and its decay is given by the probability of having just one offspring in many generations, which is exponential with exponent $p_{1}$. The left tail corresponds to an early branching, which manifests itself in the appearance of extra $(\lambda-1)$ offspring too early. The left tail is therefore controlled by the probability of keeping the sum of $\lambda$ (rather than one) i.i.d. copies of $W$ small, which explains the exponent $p^{\lambda-1}$ governing the left tail. 


\subsection{Galton-Watson process with immigration}

The remarkable difference in fluctuations of $K$ in the Schröder and Böttcher cases is due to the fact that in the former setting the minimal tree does not grow at all, having just one offspring in every generation, whereas in the latter one the minimal tree grows exponentially. A natural question to ask is what happens if the process behaves similarly to the Galton-Watson process but its minimum grows linearly. Galton-Watson process with immigration is a natural example of such a process.

Following the definition in [2], we fix a non-degenerate offspring random variable $X$ with distribution $\left(p_{k}, k \geq 0\right)$ as before, and an immigration random variable $Y$ with distribution $\left(q_{k}\right.$ : $k \geq 0)$. We define the Galton-Watson process with immigration $\left(\mathcal{Z}_{n}: n \geq 0\right)$ recursively by setting $\mathcal{Z}_{0}=Y_{0}$ and

$$
\mathcal{Z}_{n+1}=X_{1}^{(n)}+\cdots+X_{\mathcal{Z}_{n}}^{(n)}+Y_{n+1}, \quad n \geq 0
$$

where all $X_{i}^{(n)}$ are independent and identically distributed with the same distribution as $X$, all $Y_{j}$ are independent and identically distributed with the same distribution as $Y$, and all $X_{i}^{(n)}$ and $Y_{j}$ are independent. In other words, the Galton-Watson process with immigration $\left(\mathcal{Z}_{n}\right)$ differs from the ordinary Galton-Watson process with offspring probabilities $\left(p_{k}\right)$ by the property that, in generation $n$, there is an immigration of a random number of individuals into the population governed by immigration probabilities $\left(q_{k}\right)$ and independent of the rest of the process.

As before, we assume that $p_{0}=0$. We also assume that $p_{1}>0$ as otherwise the linear effect of immigration will be negligible with respect to the exponential growth of the population. For the immigration probabilities, we assume that $q_{0}=0$ in order to avoid the extinction and sub-linear growth of the minimal tree.

We assume that

$$
\mathbb{E} X \log X<\infty \text { and } \mathbb{E} \log Y<\infty
$$

and denote $a=\mathbb{E} X$, which is finite by the first condition above and greater than one since $p_{0}=0$. It is a classical result, see [13] for example, that under the conditions above the limit

$$
\mathcal{W}=\lim _{n \rightarrow \infty} \frac{\mathcal{Z}_{n}}{a^{n}}
$$

exists and is positive almost surely.

The following logarithmic left tail asymptotic was recently computed in [7]. As $\varepsilon \downarrow 0$,

$$
\log \mathbb{P}\{\mathcal{W}<\varepsilon\} \sim-\sigma \log ^{2}(1 / \varepsilon),
$$

where

$$
\sigma=\frac{\nu \log \left(1 / p_{1}\right)}{2 \log ^{2} a}
$$

and

$$
v=\min \left\{i: q_{i}>0\right\} \geq 1
$$


is the minimal number the immigration random variable can take with positive probability. As it was natural to expect, the left tail of $\mathcal{W}$ is thinner than that of the martingale limit $W$ in the Schröder case (1.1) but thicker than that of $W$ in the Böttcher case (1.2). The above result was then generalised to multitype processes in [6].

\subsection{Main results}

The aim of this paper is to find the full (non-logarithmic) left tail asymptotics of $\mathcal{W}$ at zero and to describe the time of the first non-trivial branching

$$
\mathcal{K}=\min \left\{n: \mathcal{Z}_{n}>v(n+1)\right\}
$$

conditioned on $\mathcal{W}<\varepsilon$ in the limit as $\varepsilon \downarrow 0$. In particular, we want to compare the fluctuations of $\mathcal{K}$ around its typical growth with those for Galton-Watson processes without immigration in the Schröder and Böttcher cases.

Let $\omega$ be the function defined implicitly in a right neighbourhood of zero by

$$
\omega(\varepsilon)-\log \omega(\varepsilon)+\log \log a=\log (1 / \varepsilon) .
$$

In the sequel we will drop $\varepsilon$ in most notation and, in particular, in $\omega \equiv \omega(\varepsilon)$, if there is no risk of confusion. We will also assume that $\varepsilon$ is sufficiently small.

Theorem 1.2. As $\varepsilon \downarrow 0$,

$$
\mathbb{P}\{\mathcal{W}<\varepsilon\} \sim \exp \left\{-\sigma \omega^{2}+\omega M_{1}(\omega)-\frac{1}{2} \log \omega+M_{2}(\omega)\right\},
$$

where $M_{1}$ and $M_{2}$ are bounded functions periodic with respect to $\omega$ with period $\log a$.

Remark 3. The leading term of the asymptotics (1.6) agrees with the logarithmic asymptotics (1.4) since $\omega(\varepsilon) \sim \log (1 / \varepsilon)$ according to (1.5).

Remark 4. It is of course possible to express the asymptotics of $\mathbb{P}\{\mathcal{W}<\varepsilon\}$ in terms of $\varepsilon$ rather than $\omega$ but the formula would be too bulky and less transparent, and would require replacing $\omega$ by its asymptotic decomposition in $\varepsilon$ up to the fifth order term.

Now we turn our attention to the first branching time $\mathcal{K}$. Similarly to the immigration-free case, the second largest number of offspring

$$
\lambda=\min \left\{i>1: p_{i}>0\right\}
$$

will play an important role in describing the behaviour of $\mathcal{K}$. It turns out that, conditionally on $\mathcal{W}<\varepsilon$, the typical growth of $\mathcal{K}$ is given by the scaling function $\gamma \equiv \gamma(\varepsilon)$ defined in a right neighbourhood of zero by

$$
\gamma(\varepsilon)=\frac{\log (1 / \varepsilon)}{\log a}+\left[\frac{1}{\log a}+\frac{1}{(\lambda-1) \log p_{1}}\right] \log \log (1 / \varepsilon) .
$$


The next theorem identifies $\gamma$ as the right scale and describes the fluctuations of $\mathcal{K}$ around it conditionally on $\mathcal{W}<\varepsilon$.

Theorem 1.3. There are constants $c_{1}, c_{2}>0$ such that

$$
\begin{gathered}
\underset{\varepsilon \downarrow 0}{\limsup } \mathbb{P}\{\mathcal{K}>\lfloor\gamma(\varepsilon)\rfloor+x \mid \mathcal{W}<\varepsilon\}=\exp \left\{-c_{1} p_{1}^{-(\lambda-1) x}\right\}, \\
\underset{\varepsilon \downarrow 0}{\liminf } \mathbb{P}\{\mathcal{K}>\lfloor\gamma(\varepsilon)\rfloor+x \mid \mathcal{W}<\varepsilon\}=\exp \left\{-c_{2} p_{1}^{-(\lambda-1) x}\right\}
\end{gathered}
$$

for all $x \in \mathbb{Z}$.

Remark 5. Comparing $\gamma$ with $\gamma_{\mathrm{s}}$ in the Schröder case it is easy to see that the immigration can both force the process to start branching earlier and later. The former situation occurs if $a p_{1}^{\lambda-1}>1$ and the latter if $a p_{1}^{\lambda-1}<1$. This is not intuitively clear at the first glance as we would expect an earlier branching for small values of $p_{1}$. However, the catch is that an early branching would require suppressing a larger number of the subtrees to ensure $\mathcal{W}<\varepsilon$, which is hard if $p_{1}$ is small.

Remark 6. Theorem 1.3 describes the fluctuations for every finite $x \in \mathbb{Z}$ rather than just identifying the tail behaviour as $x \rightarrow \pm \infty$. It immediately implies that the fluctuations are of finite order with a double-exponential right tail. This puts the Galton-Watson process with immigration strictly between the Böttcher case for the standard Galton-Watson process, where there are no fluctuations at all, and the Schröder case, where the right tail decays exponentially by Theorem 1.1. At the same time, the left tail still has an exponential decay since

$$
1-\exp \left\{-c_{i} p_{1}^{-(\lambda-1) x}\right\} \asymp p_{1}^{-(\lambda-1) x}
$$

as $x \rightarrow-\infty$ for $i=1,2$. Moreover, according to Theorem 1.1, the exponent of decay of the left tail is exactly the same as in the Schröder case, which shows that, unlike the right tail, the left tail is not affected by the immigration.

The comparison between the fluctuations in all three cases is given in Figure 1.

\subsection{Ideas of the proofs}

It is well known that the left tail of a positive random variable at zero is closely related to its Laplace transform at infinity. Following the approach suggested in [10], we use the precise inversion formula to obtain $\mathbb{P}\{\mathcal{W}<\varepsilon\}$ from the Laplace transform $\varphi_{*}(z)=\mathbb{E} e^{z \mathcal{W}}$. This is a technically challenging step but, unlike the standard large deviations techniques, it is capable of providing the full asymptotics of $\mathbb{P}\{\mathcal{W}<\varepsilon\}$ rather than the logarithmic one. Then we use the immigration mechanism to relate $\varphi_{*}(z)$ with the Laplace transform $\varphi(z)=\mathbb{E} e^{z W}$ of the immigration-free Galton-Watson process. Further, we rely on the well-known Poincaré functional equation

$$
\varphi\left(z a^{n}\right)=f_{n}(\varphi(z)),
$$



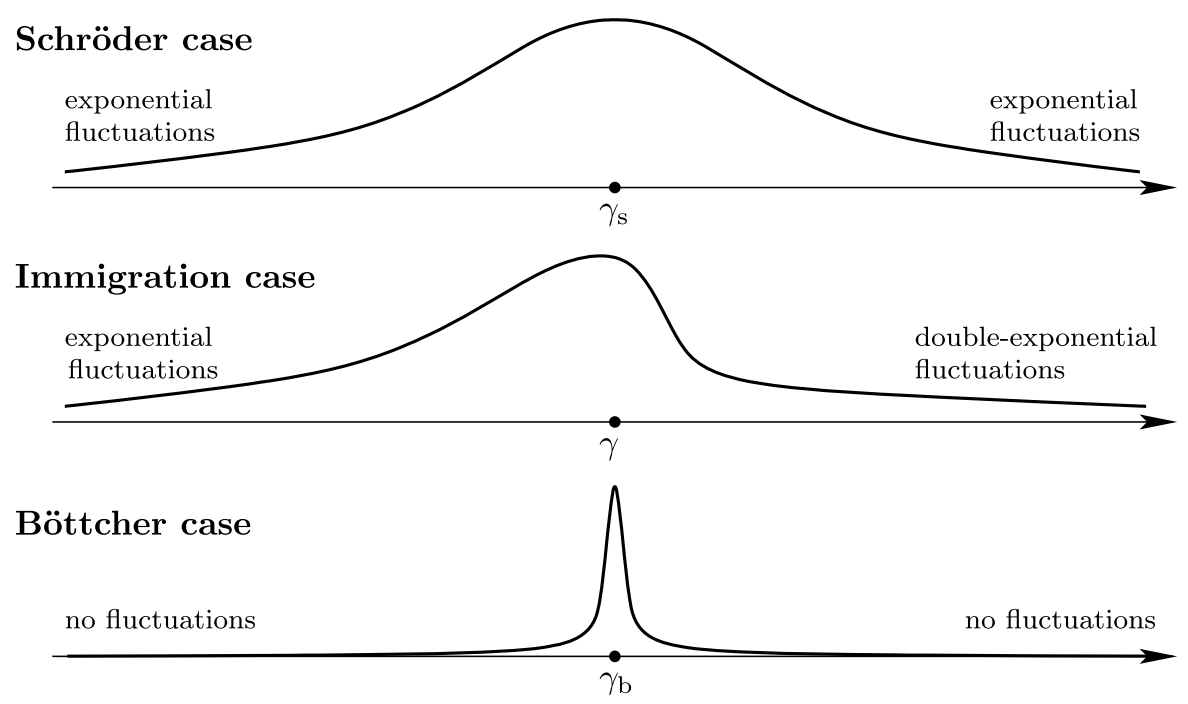

Figure 1. Tail behaviour of $\mathcal{K}$.

where $f_{n}$ is the $n$th iterate of the generating function of the offspring random variable $X$, in order to understand the behaviour of $\varphi(z)$ for large $z$ through the asymptotics of $f_{n}$ as $n$ tends to infinity.

The paper is organised as follows. In Section 2, we introduce notation and prove a couple of technical results for standard Galton-Watson processes. In Section 3, we establish the error term of the asymptotics (1.1) and use it to prove Theorem 1.1. In Section 4, we introduce notation relevant to immigration and get some preliminary asymptotic results for Galton-Watson processes with immigration. In Section 5, we prove two technical lemmas describing the behaviour of the Schröder function. In Section 6, we reduce the problem of describing the behaviour of $\mathcal{W}$ and $\mathcal{K}$ to that of understanding the left tails of a certain sequence of random variables $\left(\mathcal{V}_{n}\right)$, and establish some asymptotic properties of their Laplace transforms. In Section 7 we study the left tails of $\left(\mathcal{V}_{n}\right)$ and, finally, in Section 8 we combine everything and prove Theorems 1.2 and 1.3.

\section{Notation and preliminaries in immigration-free case}

For any $r>0$, let $\mathcal{D}_{r}=\{z \in \mathbb{C}:|z| \leq r\}$ be the closed disc of radius $r$. Denote by

$$
f(z)=\sum_{n=1}^{\infty} p_{n} z^{n}, \quad z \in \mathcal{D}_{1},
$$

the generating function of the offspring random variable $X$. Denote by

$$
\varphi(z)=\mathbb{E} e^{-z W}, \quad z \in \mathbb{C}, \mathcal{R} e z \geq 0,
$$


the Laplace transform of the martingale limit $W$ of the underlying Galton-Watson process.

Let $f_{0}(z)=z$ and, for each $n \geq 1$, denote $f_{n}(z)=f\left(f_{n-1}(z)\right)$. Since the behaviour of the iterates of $f(z)$ for large $n$ is mainly determined by its leading term $p_{1} z$, it is convenient to use the decomposition

$$
f_{n}(z)=f\left(f_{n-1}(z)\right)=p_{1} f_{n-1}(z)\left(1+p_{1}^{-1} \sum_{l>1} p_{l} f_{n-1}^{l-1}(z)\right)=p_{1}^{n} z \prod_{j=0}^{n-1} A_{j}(z),
$$

where the functions $A_{j}$ are defined on $\mathcal{D}_{1}$ by

$$
A_{j}(z)=1+p_{1}^{-1} \sum_{l>1} p_{l} f_{j}^{l-1}(z)
$$

For each $|z|<1$, denote

$$
S(z)=z \prod_{j=0}^{\infty} A_{j}(z)
$$

It is well-known (see [1], Lemma 3.7.2 and Corollary 3.7.3) that this infinite product converges uniformly on each $\mathcal{D}_{r}, r \in(0,1)$, and the function $S$ is called the Schröder function. It is easy to see that

$$
S(z)=\lim _{n \rightarrow \infty} \frac{f_{n}(z)}{p_{1}^{n}} .
$$

In particular, on each $\mathcal{D}_{r}, r \in(0,1)$, the Schröder function $S$ is bounded and $A_{j}(z) \rightarrow 1$ uniformly on $\mathcal{D}_{r}$ as $j \rightarrow \infty$. Denote

$$
\mathcal{B}=\{z \in \mathbb{C}:|z|<1, S(z) \neq 0\}
$$

For each $n \geq 0$ and $|z|<1$, denote

$$
R_{n}(z)=S(z)-p_{1}^{-n} f_{n}(z)
$$

The asymptotic behaviour of $R_{n}$ will be crucial for our analysis. In the remaining part of this section we prove some elementary properties of the functions $S, R_{n}$, and $A_{n}$.

\section{Lemma 2.1.}

(a) $|S(z)| \leq S(|z|)$ for any $|z|<1$.

(b) The functions $s \mapsto S(s)$ and $s \mapsto S(s) / s$ are increasing on $[0,1)$.

Proof. (a) This follows from the same property for each $A_{j}$ which, in turn, follows from the same property for each $f_{j}$.

(b) Obviously, it suffices to prove the second statement only. Observe that $S(s) / s$ is a product of $A_{j}(s)$, and each $A_{j}$ is increasing since each $f_{j}$ is increasing. 
For two complex-valued sequences $\left(a_{n}\right)$ and $\left(b_{n}\right)$, where $b_{n} \neq 0$ eventually, we say that $a_{n} \sim$ $b_{n}$ if $\lim _{n \rightarrow \infty} \frac{a_{n}}{b_{n}}=1$.

\section{Lemma 2.2.}

(a) Let $r \in(0,1)$. Then there are constants $c_{1}, c_{2}>0$ such that

$$
A_{n}(z)-1 \sim c_{1} S(z)^{\lambda-1} p_{1}^{n(\lambda-1)}
$$

and

$$
R_{n}(z) \sim c_{2} S^{\lambda}(z) p_{1}^{n(\lambda-1)}
$$

as $n \rightarrow \infty$ uniformly on $\mathcal{D}_{r} \cap \mathcal{B}$.

(b) $R_{n}(z)=0$ if $z \in \mathcal{D}_{r} \backslash \mathcal{B}$ for all sufficiently large $n$.

Proof. (a) Every convergence and equivalence mentioned in the proof below is meant to be uniform on $\mathcal{D}_{r} \cap \mathcal{B}$.

Using (2.1) and (2.3), we obtain

$$
R_{n}(z)=p_{1}^{-n} f_{n}(z)\left(\prod_{k=n}^{\infty} A_{k}(z)-1\right)
$$

Since $A_{j}(z) \rightarrow 1$ as $j \rightarrow \infty$ and using (2.4) we have

$$
R_{n}(z) \sim S(z)\left[\exp \left\{\sum_{j=n}^{\infty} \log A_{j}(z)\right\}-1\right] .
$$

Observe that $A_{j}(z) \neq 1$ eventually on $\mathcal{B}$. Indeed, it follows from (2.4) that $f_{n}(z) \rightarrow 0$ as $n \rightarrow \infty$. Hence the first term of the sum in (2.2) dominates over the remaining terms

$$
\left|\sum_{l>\lambda} p_{l} f_{j}^{l-1}(z)\right| \leq\left|f_{j}^{\lambda}(z)\right|=o\left(\left|f_{j}^{\lambda-1}(z)\right|\right) .
$$

It remains to notice that $f_{n}(z) \neq 0$ eventually by (2.4) and use (2.2) together with (2.7).

Now the first statement follows from

$$
\log A_{j}(z) \sim A_{j}(z)-1 \sim \frac{p_{\lambda}}{p_{1}} f_{j}^{\lambda-1}(z) \sim p_{\lambda} p_{1}^{j(\lambda-1)-1} S(z)^{\lambda-1}
$$

with $c_{1}=p_{\lambda} / p_{1}$, where the middle equivalence is implied by (2.7) and the last one by (2.4). Now we have

$$
\sum_{j=n}^{\infty} \log A_{j}(z) \sim \sum_{j=n}^{\infty} p_{\lambda} p_{1}^{j(\lambda-1)-1} S(z)^{\lambda-1} \sim c_{2} S(z)^{\lambda-1} p_{1}^{n(\lambda-1)}
$$

with some $c_{2}>0$. Substituting this into (2.6) we obtains the required asymptotics. 
(b) It is easy to see from (2.3) that $S(z)=0$ implies $z=0$ or $A_{j}(z)=0$ for some $j$. Then $f_{n}(z)=0$ eventually by $(2.1)$ and so $R_{n}(z)=0$ as well.

\section{Fluctuations in the Schröder case}

In this section, we prove Theorem 1.1. The proof for the right tail of $K-\gamma_{\mathrm{s}}$ will be rather straightforward. The left tail, however, is controlled by the second term of the asymptotics of $\mathbb{P}\{W<\varepsilon\}$, which we estimate in the proposition below.

Proposition 3.1. In the Schröder case,

$$
\begin{aligned}
& \mathbb{P}\{W<\varepsilon\}=L(\varepsilon) \varepsilon^{\tau}-\hat{L}(\varepsilon) \varepsilon^{\tau \lambda}+o\left(\varepsilon^{\tau \lambda}\right), \\
& L(\varepsilon) \varepsilon^{\tau}-\mathbb{P}\{W<\varepsilon\} \asymp \varepsilon^{\tau \lambda},
\end{aligned}
$$

as $\varepsilon \downarrow 0$, where $\tau=-\frac{\log p_{1}}{\log a}$, and $L$ and $\hat{L}$ are multiplicatively periodic positive functions on $(0, \infty)$ with period $a$. The function $L$ is analytic and the function $\hat{L}$ is bounded away from zero and infinity for all sufficiently small $\varepsilon$.

Proof. Since $W$ is almost surely positive and has no atoms, its left tail can be computed by the inversion formula

$$
\mathbb{P}\{W<\varepsilon\}=\frac{1}{2 \pi} \int_{-\infty}^{\infty} \frac{1-e^{-i \tau \varepsilon}}{i \tau} \varphi(-i \tau) d \tau .
$$

Recall the definition (1.3) of $\gamma_{\mathrm{s}}$. Changing the integration contour from the vertical axis to the vertical line passing through $a^{\left\lfloor\gamma_{\mathrm{s}}\right\rfloor}$ and substituting $\tau=t a^{\left\lfloor\gamma_{\mathrm{s}}\right\rfloor}$ we obtain

$$
\mathbb{P}\{W<\varepsilon\}=\frac{1}{2 \pi} \int_{-\infty}^{\infty} \frac{e^{\varepsilon a^{\left\lfloor\gamma_{\mathrm{s}}\right\rfloor}(1-i t)}-1}{1-i t} \varphi\left((1-i t) a^{\left\lfloor\gamma_{\mathrm{s}}\right\rfloor}\right) d t .
$$

By the definition of $\gamma_{\mathrm{s}}$ we have $\varepsilon a^{\left\lfloor\gamma_{\mathrm{s}}\right\rfloor}=a^{-\left\{\gamma_{\mathrm{s}}\right\}}$. Further, the Poincaré functional equation (1.10) and (2.5) imply

$$
\varphi\left((1-i t) a^{\left\lfloor\gamma_{\mathrm{s}}\right\rfloor}\right)=f_{\left\lfloor\gamma_{\mathrm{s}}\right\rfloor}(\varphi(1-i t))=p_{1}^{\left\lfloor\gamma_{\mathrm{s}}\right\rfloor} S(\varphi(1-i t))-p_{1}^{\left\lfloor\gamma_{\mathrm{s}}\right\rfloor} R_{\left\lfloor\gamma_{\mathrm{s}}\right\rfloor}(\varphi(1-i t))
$$

Substituting this into (3.2) and taking into account that $p_{1}^{\left\lfloor\gamma_{\mathrm{s}}\right\rfloor}=\varepsilon^{\tau} p_{1}^{-\left\{\gamma_{\mathrm{s}}\right\}}$ by the definition of $\tau$, we obtain

$$
\mathbb{P}\{W<\varepsilon\}=\varepsilon^{\tau} \frac{p_{1}^{-\left\{\gamma_{\mathrm{s}}\right\}}}{2 \pi} \int_{-\infty}^{\infty} \frac{e^{a^{-\left\{\gamma_{\mathrm{s}}\right\}}(1-i t)}-1}{1-i t}\left[S(\varphi(1-i t))-R_{\left\lfloor\gamma_{\mathrm{s}}\right\rfloor}(\varphi(1-i t))\right] d t .
$$

This representation naturally splits the left tail probability into the leading term corresponding to $S$ and the error term corresponding to $R_{\left\lfloor\gamma_{\mathrm{s}}\right\rfloor}$. Namely, we define

$$
L(\varepsilon)=\frac{p_{1}^{-\left\{\gamma_{\mathrm{s}}\right\}}}{2 \pi} \int_{-\infty}^{\infty} \frac{e^{a^{-\left\{\gamma_{\mathrm{s}}\right\}}(1-i t)}-1}{1-i t} S(\varphi(1-i t)) d t .
$$


In order to prove that the integral is finite, we first observe that the ratio under the integral is bounded. Then we get by Lemma 2.1

$$
|S(\varphi(1-i t))| \leq S(|\varphi(1-i t)|) \leq \frac{S(\varphi(1))}{\varphi(1)}|\varphi(1-i t)|
$$

and use [10], Lemma 16, which claims that $|\varphi(1-i t)|$ is integrable over $\mathbb{R}$ with respect to $t$. Hence the function $L$ is well-defined, bounded, and multiplicatively periodic with period $a$ since $\gamma_{\mathrm{S}}(\varepsilon)-\gamma_{\mathrm{s}}(a \varepsilon)=1 \in \mathbb{Z}$. In particular, once we have shown that the error term is negligible, it will imply that the function $L$ must be the same as in (1.1) and hence real-valued and is analytic.

For the error term, we use Lemma 2.2 with $r=\varphi(1)$ to get

$$
L(\varepsilon) \varepsilon^{\tau}-\mathbb{P}\{W<\varepsilon\}=\varepsilon^{\tau} \frac{p_{1}^{-\left\{\gamma_{\mathrm{s}}\right\}}}{2 \pi} \int_{-\infty}^{\infty} \frac{e^{a^{-\left\{\gamma_{\mathrm{s}}\right\}}(1-i t)}-1}{1-i t} R_{\left\lfloor\gamma_{\mathrm{s}}\right\rfloor}(\varphi(1-i t)) d t \sim \hat{L}(\varepsilon) \varepsilon^{\tau \lambda},
$$

where

$$
\hat{L}(\varepsilon)=\frac{c_{2}}{2 \pi} p_{1}^{-\lambda\left\{\gamma_{s}\right\}} \mathfrak{R e}\left[\int_{-\infty}^{\infty} \frac{e^{a^{-\{\gamma s}(1-i t)}-1}{1-i t} S^{\lambda}(\varphi(1-i t)) d t\right] .
$$

Observe that $\hat{L}$ is multiplicatively periodic with period $a$ since $\gamma_{\mathrm{s}}(\varepsilon)-\gamma_{\mathrm{s}}(a \varepsilon)=1$. It remains to show that it is positive and bounded away from zero and infinity.

To do so, consider $\lambda$ independent random variables $W_{1}, \ldots, W_{\lambda}$ with the same distribution as $W$. Observe that

$$
\begin{aligned}
\varepsilon^{\lambda \tau} & \asymp \mathbb{P}\left\{W_{1}<\varepsilon / \lambda, \ldots, W_{\lambda}<\varepsilon / \lambda\right\} \leq \mathbb{P}\left\{W_{1}+\cdots+W_{\lambda}<\varepsilon\right\} \\
& \leq \mathbb{P}\left\{W_{1}<\varepsilon, \ldots, W_{\lambda}<\varepsilon\right\} \asymp \varepsilon^{\lambda \tau}
\end{aligned}
$$

Similarly to (3.1), (3.2), (3.3), and (3.4) we have

$$
\begin{aligned}
& \mathbb{P}\left\{W_{1}+\cdots+W_{\lambda}<\varepsilon\right\} \\
& \quad=\frac{1}{2 \pi} \int_{-\infty}^{\infty} \frac{1-e^{-i \tau \varepsilon}}{i \tau} \varphi^{\lambda}(-i \tau) d \tau \\
& \quad \sim \varepsilon^{\lambda \tau} \frac{p_{1}^{-\lambda\left\{\gamma_{\mathrm{s}}\right\}}}{2 \pi} \int_{-\infty}^{\infty} \frac{e^{a^{-\left\{\gamma_{\mathrm{s}}\right\}}(1-i t)}-1}{1-i t}\left[S(\varphi(1-i t))-R_{\left\lfloor\gamma_{\mathrm{s}}\right\rfloor}(\varphi(1-i t))\right]^{\lambda} d t .
\end{aligned}
$$

By Lemma 2.2 and boundedness of $S$ we have, for all $1 \leq j \leq \lambda$,

$$
\begin{aligned}
& \left|\int_{-\infty}^{\infty} \frac{e^{a^{-\left\{\gamma_{\mathrm{s}}\right\}}(1-i t)}-1}{1-i t} S^{\lambda-j}(\varphi(1-i t)) R_{\left\lfloor\gamma_{\mathrm{s}}\right\rfloor}^{j}(\varphi(1-i t)) d t\right| \\
& \quad=O\left(\varepsilon^{\tau j(\lambda-1)}\right) \int_{-\infty}^{\infty}\left|S^{\lambda-j+\lambda j}(\varphi(1-i t))\right| d t .
\end{aligned}
$$


Since $S$ is bounded and $\lambda-j+\lambda j \geq 1$, the integral on the right-hand side is finite by the same argument as above in (3.6) combined with [10], Lemma 16. Since $\tau j(\lambda-1)>0$, this implies that the expression on the left-hand side is $o(1)$ for any $j$. Hence, the main term of the asymptotics (3.8) is given by

$$
\mathbb{P}\left\{W_{1}+\cdots+W_{\lambda}<\varepsilon\right\} \sim \varepsilon^{\lambda \tau} \frac{p_{1}^{-\lambda\left\{\gamma_{\mathrm{s}}\right\}}}{2 \pi} \int_{-\infty}^{\infty} \frac{e^{a^{-\left\{\gamma_{\mathrm{s}}\right\}}(1-i t)}-1}{1-i t} S^{\lambda}(\varphi(1-i t)) d t \sim \frac{\hat{L}(\varepsilon)}{c_{2}} \varepsilon^{\lambda \tau}
$$

since the expression on the left-hand side is real. Now (3.7) implies that $\hat{L}$ is positive and bounded away from zero and infinity eventually.

Proof of Theorem 1.1. It suffices to prove the theorem for $x \in \mathbb{Z}$. Observe that the condition $K>\gamma_{\mathrm{s}}+x$ is equivalent to having just one offspring in the generation $\left\lfloor\gamma_{\mathrm{s}}\right\rfloor+x$. On this event, the condition $W<\varepsilon$ is equivalent to $\hat{W}<a^{\left\lfloor\gamma_{s}\right\rfloor+x} \varepsilon$, where $\hat{W}$ is the martingale limit of the GaltonWatson subtree generated by that offspring. Hence, $\hat{W}$ has the same distribution as $W$ but is also independent of the event $Z_{\left\lfloor\gamma_{\mathrm{s}}\right\rfloor+x}=1$. Using (1.3), we obtain

$$
\mathbb{P}\left\{K>\gamma_{\mathrm{s}}+x, W<\varepsilon\right\}=\mathbb{P}\left\{Z_{\left\lfloor\gamma_{\mathrm{s}}\right\rfloor+x}=1, \hat{W}<a^{\left\lfloor\gamma_{\mathrm{s}}\right\rfloor+x} \varepsilon\right\}=p_{1}^{\left\lfloor\gamma_{\mathrm{s}}\right\rfloor+x} \mathbb{P}\left\{\hat{W}<a^{-\left\{\gamma_{\mathrm{s}}\right\}+x}\right\} .
$$

Combining this with the left tail asymptotics (1.1) and using $p_{1}^{\gamma_{\mathrm{s}}}=\varepsilon^{\tau}$ we get

$$
\mathbb{P}\left\{K>\gamma_{\mathrm{s}}+x \mid W<\varepsilon\right\} \sim \frac{p_{1}^{\left\lfloor\gamma_{\mathrm{s}}\right\rfloor+x}}{L(\varepsilon) \varepsilon^{\tau}} \mathbb{P}\left\{\hat{W}<a^{-\left\{\gamma_{\mathrm{s}}\right\}+x}\right\}=\frac{p_{1}^{-\left\{\gamma_{\mathrm{s}}\right\}+x}}{L\left(a^{-\left\{\gamma_{\mathrm{s}}\right\}}\right)} \mathbb{P}\left\{\hat{W}<a^{-\left\{\gamma_{\mathrm{s}}\right\}+x}\right\}
$$

as $\varepsilon \downarrow 0$ since $L$ is multiplicatively periodic with period $a$ and $\varepsilon=a^{-\gamma_{\mathrm{s}}}$. Observe that, for a fixed $x$, the expression on the right-hand side only depends on $\left\{\gamma_{\mathrm{s}}\right\}$ and so is multiplicatively periodic in $\varepsilon$. This implies that

$$
\begin{gathered}
\liminf _{\varepsilon \downarrow 0} \mathbb{P}\left\{K>\gamma_{\mathrm{s}}+x \mid W<\varepsilon\right\}=p_{1}^{x} \frac{p_{1}^{-\alpha_{1}}}{L\left(a^{-\alpha_{1}}\right)} \mathbb{P}\left\{\hat{W}<a^{-\alpha_{1}+x}\right\}, \\
\underset{\varepsilon \downarrow 0}{\lim \sup _{0}} \mathbb{P}\left\{K>\gamma_{\mathrm{s}}+x \mid W<\varepsilon\right\}=p_{1}^{x} \frac{p_{1}^{-\alpha_{2}}}{L\left(a^{-\alpha_{2}}\right)} \mathbb{P}\left\{\hat{W}<a^{-\alpha_{2}+x}\right\},
\end{gathered}
$$

for some $\alpha_{1}, \alpha_{2} \in[0,1]$. It suffices now to show that uniformly for all $\alpha \in[0,1]$

$$
p_{1}^{x} \frac{p_{1}^{-\alpha}}{L\left(a^{-\alpha}\right)} \mathbb{P}\left\{\hat{W}<a^{-\alpha+x}\right\} \asymp p_{1}^{x}
$$

and

$$
1-p_{1}^{-x} \frac{p_{1}^{-\alpha}}{L\left(a^{-\alpha}\right)} \mathbb{P}\left\{\hat{W}<a^{-\alpha-x}\right\} \asymp p_{1}^{(\lambda-1) x}
$$

as $x \rightarrow \infty$. The first identity easily follows from the fact that

$$
\mathbb{P}\left\{\hat{W}<a^{-\alpha+x}\right\} \geq \mathbb{P}\left\{\hat{W}<a^{-1+x}\right\} \rightarrow 1
$$


as $x \rightarrow \infty$. For the second one we observe that $a^{-\alpha-x} \downarrow 0$ as $x \rightarrow \infty$ and so we can derive the above asymptotics from the left tail asymptotics of the martingale limit obtained in Proposition 3.1. As $L$ is multiplicatively periodic with period $a$ and $x$ is an integer we have

$$
\begin{aligned}
1-p_{1}^{-x} \frac{p_{1}^{-\alpha}}{L\left(a^{-\alpha}\right)} \mathbb{P}\left\{\hat{W}<a^{-\alpha-x}\right\} & \asymp p_{1}^{-x}\left(L\left(a^{-\alpha-x}\right) a^{-\tau(\alpha+x)}-\mathbb{P}\left\{\hat{W}<a^{-\alpha-x}\right\}\right) \\
& \asymp p_{1}^{-x} a^{-\tau \lambda(\alpha+x)} \asymp p_{1}^{(\lambda-1) x}
\end{aligned}
$$

since $a^{-\tau}=p_{1}$.

\section{Immigration}

In this section, we introduce notation relevant to the immigration and prove some preliminary results which will be necessary to deal with it. Denote by

$$
h(z)=\sum_{n=v}^{\infty} q_{n} z^{n}, \quad z \in \mathcal{D}_{1}
$$

the generating function of the random variable $Y$. We will see in Section 6 that in order to find the left tail asymptotics of $\mathcal{W}$ we will have to control the products

$$
\prod_{n=1}^{N} h\left(f_{n}(z)\right)
$$

for $N \in \mathbb{N}$. Since $f_{n}(z)$ will typically tend to zero as $n \rightarrow \infty$, the function $h(z)$ will essentially behave according to its leading term $q_{v} z^{\nu}$. This observation suggests using the decomposition

$$
h\left(f_{n}(z)\right)=q_{v} f_{n}^{v}(z)\left(1+q_{\nu}^{-1} \sum_{l>v} q_{l} f_{n}^{l-v}(z)\right)=q_{v} f_{n}^{v}(z) B_{n}(z),
$$

where the functions $B_{n}$ are defined by

$$
B_{n}(z)=1+q_{v}^{-1} \sum_{l>v} q_{l} f_{n}^{l-v}(z), \quad z \in \mathcal{D}_{1} .
$$

Combining (2.1) and (4.2), we obtain

$$
h\left(f_{n}(z)\right)=q_{\nu} p_{1}^{n v} z^{v} B_{n}(z) \prod_{j=0}^{n-1} A_{j}^{v}(z) .
$$

For any $r \in(0,1)$ and $\theta \in(0, \pi / 2)$, denote

$$
\mathcal{D}_{r, \theta}=\left\{z \in \mathcal{D}_{r}: z \neq 0,|\arg z| \leq \theta\right\} .
$$


Lemma 4.1. Let $r \in(0,1)$. As $n \rightarrow \infty$,

$$
\prod_{j=1}^{n} B_{j}(z) \rightarrow B(z)
$$

uniformly on $\mathcal{D}_{r}$, where $B$ is a bounded holomorphic function.

Further, there exists $\theta \in(0, \pi / 2)$ such that on $\mathcal{D}_{r, \theta}$ :

(a) $S(z) \neq 0$ and, in particular, $A_{k}(z) \neq 0$ for all $k$;

(b) $B(z) \neq 0$;

(c)

$$
\prod_{j=1}^{n} A_{j}^{-j}(z) \rightarrow C(z) \neq 0
$$

uniformly as $n \rightarrow \infty$, where $C$ is a bounded holomorphic function.

Proof. Using (4.3) and monotonicity of $f_{n}$ as well as that it is bounded by 1 on $(0,1)$ we get

$$
\left|B_{j}(z)-1\right| \leq q_{v}^{-1} \sum_{l>v} q_{l}\left|f_{j}^{l-v}(z)\right| \leq q_{v}^{-1} f_{j}(r) \sim q_{v}^{-1} S(r) p_{1}^{j}
$$

for all $z \in \mathcal{D}_{r}$ uniformly. Since the sum over $j$ of the expressions on the right-hand side is finite, $\prod_{j=1}^{n} B_{j}(z)$ converges uniformly on $D_{r}$, and hence $B$ is holomorphic and bounded.

Let us now prove the second part of the lemma.

(a) Observe that $S$ is holomorphic on $\mathcal{D}_{r}$ as a uniform limit of holomorphic functions. Hence, it can only have isolated zeroes, and it suffices to observe that $S(s) \neq 0$ for $s \in(0,1)$ and choose $\theta$ sufficiently small.

(b) Similarly, $B$ can only have isolated zeroes, and it is easy to see from (4.3) that $B(s) \geq 1$ for $s \in[0,1]$. Hence, one can choose $\theta$ small enough so that $B$ has no zeroes on $\mathcal{D}_{r, \theta}$.

(c) First, we observe that by (a) the product is well defined for a sufficiently small $\theta$. Second, by Lemma 2.2 we know that

$$
j \log A_{j}(z) \sim c S(z)^{\lambda-1} j p_{1}^{j(\lambda-1)}
$$

uniformly as $j \rightarrow \infty$, where $S(z) \neq 0$ by (a) if $\theta$ is small enough. Again, the sum over $j$ of the expressions on the right hand side is finite, and we can apply the same arguments as for the first product.

\section{Lemma 4.2.}

(a) Let $r \in(0,1)$. There is $c>0$ such that, for all $s \in(0, r], N$ and $z \in \mathcal{D}_{s}$,

$$
\left|\prod_{n=1}^{N} h\left(f_{n}(z)\right)\right| \leq c \frac{|z|}{s} q_{\nu}^{N} p_{1}^{-\frac{v N(N+1)}{2}} f_{N+1}^{v N}(s) .
$$


(b) Let $r \in(0,1)$ and let $\theta$ be chosen according to Lemma 4.1. Then

$$
\prod_{n=1}^{N} h\left(f_{n}(z)\right) \sim F(z) q_{\nu}^{N} p_{1}^{-\frac{v N(N+1)}{2}} f_{N+1}^{\nu N}(z)
$$

as $N \rightarrow \infty$ uniformly on $\mathcal{D}_{r, \theta}$, where $F$ is a bounded function on $\mathcal{D}_{r, \theta}$, which is nowhere equal to zero.

Proof. It follows from (4.4) that, for all $N \geq 1$, we have

$$
\prod_{n=1}^{N} h\left(f_{n}(z)\right)=q_{\nu}^{N} p_{1}^{\frac{v N(N+1)}{2}} z^{\nu N}\left(\prod_{n=1}^{N} \prod_{j=0}^{n-1} A_{j}^{\nu}(z)\right)\left(\prod_{n=1}^{N} B_{n}(z)\right)
$$

(a) Observe that the last term is uniformly (in $s$ and $z$ ) bounded by Lemma 4.1. Further, it follows from (2.2) that $\left|A_{j}(z)\right| \leq A_{j}(s)$ for all $j$ on $\mathcal{D}_{s}$. Hence, for all $z \in \mathcal{D}_{s}$,

$$
\left|z^{\nu N} \prod_{n=1}^{N} \prod_{j=0}^{n-1} A_{j}^{v}(z)\right| \leq|z| s^{\nu N-1} \prod_{n=1}^{N} \prod_{j=0}^{n-1} A_{j}^{v}(s)=|z| s^{\nu N-1} \prod_{j=0}^{N} A_{j}^{\nu(N-j)}(s),
$$

where we included $j=N$ into the product since the corresponding term equals one. As all $A_{j}(s)>1$ we can drop $j$ in the exponent which together with (2.1) implies

$$
\left|z^{v N} \prod_{n=1}^{N} \prod_{j=0}^{n-1} A_{j}^{v}(z)\right| \leq \frac{|z|}{s} p_{1}^{-v N(N+1)} f_{N+1}^{v N}(s) .
$$

(b) It follows from Lemma 4.1 that all $A_{j}(z) \neq 0$ and so we can rearrange the middle product in (4.8) and use (2.1) to get

$$
z^{v N} \prod_{n=1}^{N} \prod_{j=0}^{n-1} A_{j}^{v}(z)=z^{v N} \prod_{j=0}^{N} A_{j}^{v(N-j)}(z)=p_{1}^{-v N(N+1)} f_{N+1}^{v N}(z) \prod_{j=0}^{N} A_{j}^{-v j}(z) .
$$

Now the statement of the lemma follows from Lemma 4.1 with $F(z)=B(z) C^{v}(z)$.

\section{Finer properties of the Schröder function $S$}

Lemma 5.1. The function $s \mapsto \log S(s)$ is well-defined and analytic on $(0,1)$, and

$$
(\log S(s))^{\prime} \geq 1 / s
$$

for all $s \in(0,1)$. 
Proof. Since $S(s) \geq s$ by (2.3) the logarithm is well defined in a complex neighbourhood of $s$, and the analyticity follows from $\log S$ being holomorphic there. Differentiating the uniform limit of holomorphic functions (2.4) we obtain

$$
(\log S(s))^{\prime}=\frac{S^{\prime}(s)}{S(s)}=\lim _{n \rightarrow \infty} \frac{f_{n}^{\prime}(s)}{f_{n}(s)} \geq 1 / s
$$

since this inequality is true term by term for $f_{n}^{\prime}$ and $f_{n}$.

Denote

$$
\psi(s)=\log S(\varphi(s)), \quad s>0
$$

Lemma 5.2. The function $\psi$ is analytic, $\psi^{\prime \prime}(s)>0$ for all $s$, and

$$
\lim _{s \rightarrow \infty} \psi^{\prime}(s)=0 \quad \text { and } \quad \lim _{s \downarrow 0} \psi^{\prime}(s)=-\infty .
$$

Proof. The function $\psi$ is analytic as a composition of analytic functions. Compute

$$
\begin{aligned}
\psi^{\prime}(s) & =\frac{\varphi^{\prime}(s) S^{\prime}(\varphi(s))}{S(\varphi(s))} \\
\psi^{\prime \prime}(s) & =\frac{\left(\varphi^{\prime \prime}(s) S^{\prime}(\varphi(s))+\left(\varphi^{\prime}(s)\right)^{2} S^{\prime \prime}(\varphi(s))\right) S(\varphi(s))-\varphi^{\prime}(s)\left(S^{\prime}(\varphi(s))\right)^{2}}{S^{2}(\varphi(s))} .
\end{aligned}
$$

It was shown in [10], (75), that $\varphi^{\prime \prime}(s) \varphi(s)>\left(\varphi^{\prime}(s)\right)^{2}$ for all $s>0$. Further, $S$ is positive according to (2.3) and $S^{\prime}$ is positive by Lemma 5.1. This implies

$$
\psi^{\prime \prime}(s)>\frac{\left(S^{\prime}(\varphi(s))+\varphi(s) S^{\prime \prime}(\varphi(s))\right)\left(\varphi^{\prime}(s)\right)^{2} S(\varphi(s))-\varphi(s) \varphi^{\prime}(s)\left(S^{\prime}(\varphi(s))\right)^{2}}{S^{2}(\varphi(s)) \varphi(s)} .
$$

Using [10], (63), for $f_{n}$ instead of $f$ we obtain

$$
\left(\frac{s f_{n}^{\prime}(s)}{f_{n}(s)}\right)^{\prime}>0
$$

for all $s \in(0,1)$ and all $n$, which extends to

$$
\left(\frac{s S^{\prime}(s)}{S(s)}\right)^{\prime} \geq 0
$$

for all $s \in(0,1)$ since one can differentiate uniformly convergent series of analytic functions. This implies

$$
\left(S^{\prime}(s)+s S^{\prime \prime}(s)\right) S(s) \geq s\left(S^{\prime}(s)\right)^{2}
$$


for all $s \in(0,1)$. Substituting this into (5.2), we get

$$
\psi^{\prime \prime}(s)>\frac{\left(\varphi^{\prime}(s)-1\right) \varphi^{\prime}(s)\left(S^{\prime}(\varphi(s))\right)^{2}}{S^{2}(\varphi(s))} \geq 0
$$

for all $s>0$ since $\varphi^{\prime}(s) \leq 0$.

To prove (5.1), we observe that by the Poincaré functional equation and (2.4) we have

$$
\psi\left(s a^{n}\right)=\log S\left(f_{n}(\varphi(s))\right)=\log \left(p_{1}^{n} S(\varphi(s))\right)=\psi(s)+n \log p_{1}
$$

and so

$$
a^{n} \psi^{\prime}\left(s a^{n}\right)=\psi^{\prime}(s)
$$

for all $s>0$ and all $n$. Since $\psi^{\prime \prime}$ is positive $\psi^{\prime}$ is decreasing and so

$$
\begin{aligned}
& \lim _{s \rightarrow \infty} \psi^{\prime}(s)=\lim _{n \rightarrow \infty} \psi^{\prime}\left(a^{n}\right)=\psi^{\prime}(1) \lim _{n \rightarrow \infty} a^{-n}=0, \\
& \lim _{s \downarrow 0} \psi^{\prime}(s)=\lim _{n \rightarrow \infty} \psi^{\prime}\left(a^{-n}\right)=\psi^{\prime}(1) \lim _{n \rightarrow \infty} a^{n}=-\infty,
\end{aligned}
$$

as required.

\section{The random variables $\mathcal{V}_{n}$ and their Laplace transforms}

Denote by

$$
\varphi_{*}(z)=\mathbb{E} e^{-z \mathcal{W}}, \quad z \in \mathbb{C}, \mathcal{R} e z \geq 0,
$$

the Laplace transform of the random variable $\mathcal{W}$. Observe that the $m$ th generation of the GaltonWatson tree with immigration can be written as

$$
\mathcal{Z}_{n}=\sum_{i=1}^{Y_{0}} Z_{n}^{(i)}+\hat{\mathcal{Z}}_{n-1}
$$

where where $Z^{(i)}$ is the Galton-Watson process corresponding to the $i$ th immigrant in the generation zero, and $\hat{\mathcal{Z}}$ is the Galton-Watson process with immigration starting with the immigrants of generation one. It is easy to see that the process $\hat{\mathcal{Z}}$ and all processes $Z^{(i)}$ are independent, and $\hat{\mathcal{Z}}$ has the same distribution as $\mathcal{Z}$. Dividing by $a^{n}$ and passing to the limit we obtain

$$
\mathcal{W}=\sum_{i=1}^{Y_{0}} W_{i}+a^{-1} \hat{\mathcal{W}}
$$

where $W_{i}$ are the martingale limits of the processes $Z^{(i)}$, and $\hat{\mathcal{W}}$ is the limit random variable corresponding to $\hat{\mathcal{Z}}$. Clearly, $\hat{\mathcal{W}}$ and all $W_{i}$ are independent and have the same distribution as $\mathcal{W}$ 
and $W$, respectively. This implies

$$
\varphi_{*}(z)=h(\varphi(z)) \varphi_{*}\left(z a^{-1}\right) .
$$

Further, for any $k \geq-1$, denote by

$$
\mathcal{V}_{k}=a^{-k} \sum_{i=1}^{\nu(k+1)} W_{i}^{(k)}+a^{-k-1} \hat{\mathcal{W}}^{(k)},
$$

where $W_{i}^{(k)}, 1 \leq i \leq v(k+1)$, are the martingale limits of independent Galton-Watson processes indexed by the first $v$ immigrants of all generations between 0 and $k$, and $\hat{\mathcal{W}}^{(k)}$ is the limit random variable of the independent Galton-Watson process with immigration starting with the immigrants of generations strictly after $k$. Similarly to (6.3) we observe that

$$
\mathcal{W}=\mathcal{V}_{k} \quad \text { on the event }\{\mathcal{K}>k\}
$$

since the minimal number of individuals in generation $k$ is $v(k+1)$ given by $v$ immigrants in each generation and having just one offspring each. Denote the Laplace transform of $\mathcal{V}_{k}$ by

$$
\varphi_{k}(z)=\mathbb{E} e^{-z \mathcal{V}_{k}}, \quad z \in \mathbb{C}, \mathcal{R} e z \geq 0 .
$$

It follows from (6.5) that

$$
\varphi_{k}(z)=\varphi\left(z a^{-k}\right)^{v(k+1)} \varphi_{*}\left(z a^{-k-1}\right) .
$$

Observe that $\mathcal{V}_{-1}=\mathcal{W}$ and $\varphi_{-1}=\varphi_{*}$. We also denote $Z_{-1}=0$.

For each $k \geq-1$ we have

$$
\mathbb{P}\{\mathcal{K}>k, \mathcal{W}<\varepsilon\}=\mathbb{P}\left\{Z_{k}=v(k+1), \mathcal{V}_{k}<\varepsilon\right\}=q_{\nu}^{k+1} p_{1}^{\frac{\nu k(k+1)}{2}} \mathbb{P}\left\{\mathcal{V}_{k}<\varepsilon\right\}
$$

This means that our main aim now is to understand the left tail probabilities of $\mathcal{V}_{k}$. Those corresponding to $k=-1$ will give us the left tail asymptotics of $\mathcal{W}$, and those with $k=\gamma(\varepsilon)+x$ will control the fluctuations. Recall that the left tail of $\mathcal{V}_{k}$ is closely related to the behaviour of the Laplace transform $\varphi_{k}$ for large values of the argument. The next lemma enables us to understand it through the asymptotic properties of the iterations $f_{n}$ and the immigration mechanism $h$ in the same spirit as the Poincaré functional equation does it for a standard Galton-Watson process.

For any $k$ and $N$, denote

$$
C_{k, N}=q_{v}^{N-k-1} p_{1}^{\frac{v N(N+1)-v k(k+1)}{2}}
$$

Further, for any $z \in \mathcal{B}$, denote

$$
\Psi_{k, N}(z)=\left(1-\frac{R_{N-k}(z)}{S(z)}\right)^{v N} .
$$




\section{Lemma 6.1.}

(a) Let $r \in(0,1)$. There is $c>0$ such that

$$
\left|\varphi_{k}\left(z a^{N}\right)\right| \leq c \frac{|\varphi(z)|}{s} C_{k, N} S(s)^{v N}
$$

for all $k<N, s \in(0, r]$, and all $z$ satisfying $|\varphi(z)| \leq s$.

(b) Let $r \in(0,1)$ and let $\theta$ be chosen according to Lemma 4.1. Then

$$
\varphi_{k}\left(z a^{N}\right) \sim \Psi_{k, N}(\varphi(z)) \varphi_{*}(z) F(\varphi(z)) C_{k, N} S(\varphi(z))^{v N}
$$

as $N-k \rightarrow \infty$ uniformly on $\left\{z: \varphi(z) \in \mathcal{D}_{r, \theta}\right\}$.

Proof. Using (6.6) and iterating it according to (6.4), we obtain

$$
\varphi_{k}\left(z a^{N}\right)=\varphi_{*}(z) \varphi\left(z a^{N-k}\right)^{v(k+1)} \prod_{n=1}^{N-k-1} h\left(\varphi\left(z a^{n}\right)\right) .
$$

The Poincaré functional equation (1.10) implies

$$
\varphi_{k}\left(z a^{N}\right)=\varphi_{*}(z) f_{N-k}^{\nu(k+1)}(\varphi(z)) \prod_{n=1}^{N-k-1} h\left(f_{n}(\varphi(z))\right) .
$$

(a) Estimating the Laplace transform $\varphi_{*}$ by one, the second term of (6.10) by

$$
\left|f_{N-k}(\varphi(z))\right| \leq f_{N-k}(|\varphi(z)|) \leq f_{N-k}(s),
$$

and the product by Lemma 4.2 we have

$$
\left|\varphi_{k}\left(z a^{N}\right)\right| \leq c \frac{|\varphi(z)|}{s} q_{\nu}^{N-k-1} p_{1}^{-\frac{\nu(N-k-1)(N-k)}{2}} f_{N-k}^{\nu N}(s)
$$

with some $c>0$. Taking into account

$$
f_{N-k}(s) \leq p_{1}^{N-k} S(s),
$$

which follows from (2.1) and (2.3) we obtain the required bound.

(b) Using the asymptotics for the product in (6.10) obtained in Lemma 4.2 we get

$$
\varphi_{k}\left(z a^{N}\right) \sim \varphi_{*}(z) F(\varphi(z)) q_{\nu}^{N-k-1} p_{1}^{-\frac{\nu(N-k-1)(N-k)}{2}} f_{N-k}^{\nu N}(\varphi(z))
$$

as $N-k \rightarrow \infty$ uniformly on $\left\{z: \varphi(z) \in \mathcal{D}_{r, \theta}\right\}$. Taking into account

$$
f_{N-k}(\varphi(z))=p_{1}^{N-k} S(\varphi(z))-p_{1}^{N-k} R_{N-k}(\varphi(z))
$$

and observing that $S(\varphi(z)) \neq 0$ on $\left\{z: \varphi(z) \in \mathcal{D}_{r, \theta}\right\}$ by Lemma 4.1 we arrive at (6.9). 


\section{Left tail of $\mathcal{V}_{k}$}

The aim of this section is to compute the left tail asymptotics for $\mathcal{V}_{k}$ for two types of $k$. The first case is simply

$$
k=-1 \text {. }
$$

Combined with (6.7), this would give us the left tail asymptotics of $\mathcal{W}$ and prove Theorem 1.2. The second case is

$$
k \equiv k(\varepsilon, x)=\lfloor\gamma(\varepsilon)\rfloor+x
$$

for a fixed integer $x$. This is needed to prove Theorem 1.3. It turns out that both cases can be handled simultaneously so in this section we always assume that $k$ satisfies either (7.1) or (7.2).

Let $\rho$ be the function defined implicitly in a right neighbourhood of zero by

$$
\rho(\varepsilon) a^{-\rho(\varepsilon)}=\varepsilon
$$

It is easy to see that

$$
\omega=\rho \log a .
$$

and that the first three leading terms ${ }^{1}$ of the asymptotics of $\rho$ are given by

$$
\rho(\varepsilon)=\frac{1}{\log a}[\log (1 / \varepsilon)+\log \log (1 / \varepsilon)-(1+o(1)) \log \log a] .
$$

It is worth mentioning that the definition (1.7) of $\gamma$ manifested itself from the condition

$$
\rho(\varepsilon) p_{1}^{(\lambda-1)(\rho(\varepsilon)-\gamma(\varepsilon))} \asymp 1 \quad \text { as } \varepsilon \downarrow 0,
$$

which will prove to be crucial later on.

Now fix

$$
N \equiv N(\varepsilon)=\lfloor\rho(\varepsilon)\rfloor .
$$

and choose $u \equiv u(\varepsilon)$ in such a way that

$$
\nu \psi^{\prime}(u)=-a^{-\{\rho\}} .
$$

This is possible by Lemma 5.2 since $\psi^{\prime}$ takes all negative values. Moreover, since the right hand side of (7.7) is bounded between -1 and $-1 / a$ and $\psi^{\prime}$ is decreasing by Lemma 5.2, there

\footnotetext{
${ }^{1}$ We only need the three leading terms to understand the left tail asymptotics of $\mathcal{V}_{k}$. However, one can easily obtain the more precise asymptotics

$$
\omega(\varepsilon)=\log (1 / \varepsilon)+\log \log (1 / \varepsilon)-\log \log a+\frac{\log \log (1 / \varepsilon)}{\log (1 / \varepsilon)}-\frac{\log \log a}{\log (1 / \varepsilon)}+o\left(\frac{1}{\log (1 / \varepsilon)}\right)
$$

which, if substituted into (1.6), provides an explicit asymptotics of $\mathbb{P}\{\mathcal{W}<\varepsilon\}$ in terms of $\varepsilon$.
} 
exist positive and independent of $\varepsilon$ constants $u_{*}$ and $u^{*}$ such that $u \in\left[u_{*}, u^{*}\right]$ for all sufficiently small $\varepsilon$.

Similarly to (3.1), the lower tail of $\mathcal{V}_{k}$ can be computed by the inversion formula

$$
\mathbb{P}\left\{\mathcal{V}_{k}<\varepsilon\right\}=\frac{1}{2 \pi} \int_{-\infty}^{\infty} \frac{1-e^{-i \tau \varepsilon}}{i \tau} \varphi_{k}(-i \tau) d \tau
$$

Similarly to (3.2), we will move the integration contour far to the right and rescale the integration accordingly. However, we have to do it more carefully. Namely, we replace the vertical coordinate axis by the vertical line passing through $u a^{N}$ and substitute $\tau=t a^{N}$. This gives

$$
\mathbb{P}\left\{\mathcal{V}_{k}<\varepsilon\right\}=\frac{1}{2 \pi} \int_{-\infty}^{\infty} \frac{e^{\varepsilon a^{N}(u-i t)}-1}{u-i t} \varphi_{k}\left((u-i t) a^{N}\right) d t
$$

In order to understand the impact of the term $\varphi_{k}$ in the integral, we will combine Lemma 6.1 with the saddle point approximation for $S$. Choose $r$ in such a way that $\varphi\left(u_{*}\right)<r<1$ and choose $\theta$ according to Lemma 4.1. The composition $S \circ \varphi$ maps the interval $\left[u_{*}, u^{*}\right]$ to $\left[S\left(\varphi\left(u^{*}\right)\right), S\left(\varphi\left(u_{*}\right)\right)\right]$ since $\varphi$ is decreasing on $[0, \infty)$ and $S$ is increasing on $[0,1)$ by Lemma 2.1 . Hence we can choose $\beta_{1}>0$ small enough so that $\varphi$ maps $\left[u_{*}, u^{*}\right] \times\left[-\beta_{1}, \beta_{1}\right]$ to $\mathcal{D}_{r, \theta}$ and $S \circ \varphi$ maps $\left[u_{*}, u^{*}\right] \times\left[-\beta_{1}, \beta_{1}\right]$ to $\{z \in \mathbb{C}: \mathcal{R} e z>0\}$. Now the function $\log (S \circ \varphi)$ is well-defined on $\left[u_{*}, u^{*}\right] \times\left[-\beta_{1}, \beta_{1}\right]$ and its third order derivatives are bounded. Expanding into the Taylor series

$$
\log S(\varphi(u-i t))=\log S(\varphi(u))-i t \psi^{\prime}(u)-\frac{t^{2}}{2} \psi^{\prime \prime}(u)+O\left(t^{3}\right)
$$

as $t \rightarrow 0$ uniformly in $\varepsilon$ and using the fact that $\psi^{\prime \prime}$ is positive by Lemma 5.2, we choose $\beta \in$ $\left(0, \beta_{1}\right)$ in such a way that

$$
\mathcal{R} e[\log S(\varphi(u-i t))] \leq \log S(\varphi(u))-\frac{t^{2}}{4} \psi^{\prime \prime}(u)
$$

for all $t \in[-\beta, \beta]$ and all $\varepsilon$ small enough. Finally, we choose $\alpha \equiv \alpha(\varepsilon)$ so that $\alpha \downarrow 0$,

$$
\alpha e^{N \alpha^{3}} \rightarrow 0 \quad \text { and } \quad \alpha^{2} N / \log N \rightarrow \infty
$$

We assume that $\varepsilon$ is small enough so that $\alpha<\beta$. In Lemmas 7.2, 7.3, and 7.4 below we will compute the main part of the integral (7.8) coming from integrating over $[-\alpha, \alpha]$, and show that the integrals over the remaining parts $\{|t| \in[\alpha, \beta]\}$ and $\{|t|>\beta\}$ are negligible.

However, before turning our attention to the integral (7.8) we compute the asymptotics of $\Psi_{k, N}$, which plays a crucial rôle for $\varphi_{k}\left((u-i t) a^{N}\right)$ according to Lemma 6.1.

Lemma 7.1. Let $r \in(0,1)$ and let $\theta$ be chosen according to Lemma 4.1.

(a) $\Psi_{-1, N}(z) \sim 1$ as $\varepsilon \downarrow 0$ uniformly on $\mathcal{D}_{r, \theta}$. 
(b) Let $k$ be of the form (7.2). Then there is $w \equiv w(\varepsilon)$ bounded away from zero and infinity such that

$$
\Psi_{k, N}(z) \sim \exp \left\{-w \nu p_{1}^{-(\lambda-1) x} S(z)^{\lambda-1}\right\}
$$

as $\varepsilon \downarrow 0$ uniformly on $\mathcal{D}_{r, \theta}$.

Proof. Comparing the definition of $\gamma$ in (1.7) and the asymptotics (7.5) of $\rho$ it is easy to see that $N-k \rightarrow \infty$ as $\varepsilon \downarrow 0$ in both cases. Observe that $S(z) \neq 0$ on $\mathcal{D}_{r, \theta}$ by Lemma 4.1. Lemma 2.2 now implies that

$$
\frac{R_{N-k}(z)}{S(z)} \sim c_{1} S(z)^{\lambda-1} p_{1}^{(\lambda-1)(N-k)}
$$

with some $c_{1}>0$ and hence

$$
\log \Psi_{k, N} \sim-c_{1} v N S(z)^{\lambda-1} p_{1}^{(\lambda-1)(N-k)}
$$

as $\varepsilon \downarrow 0$ uniformly on $\mathcal{D}_{r, \theta}$

(a) If $k=-1$ then we use $N \sim \frac{\log (1 / \varepsilon)}{\log a}$ to obtain

$$
\lim _{\varepsilon \downarrow 0} N p_{1}^{(\lambda-1)(N+1)}=0 .
$$

(b) For $k=\gamma-\{\gamma\}+x$ we get

$$
N p_{1}^{(\lambda-1)(N-k)} \sim p_{1}^{-x(\lambda-1)} p_{1}^{(\lambda-1)(\{\gamma\}-\{\rho\})} \rho p_{1}^{(\lambda-1)(\rho-\gamma)} .
$$

Since $\rho p_{1}^{(\lambda-1)(\rho-\gamma)} \sim c_{2}$ according to (1.7) and the three leading terms of $\rho$ given by (7.5), we obtain the required asymptotics with $w \equiv w(\varepsilon)=c_{1} c_{2} p_{1}^{(\lambda-1)(\{\gamma\}-\{\rho\})}$.

Lemma 7.2. Suppose that $k$ is of the form (7.1) or (7.2). Then

$$
\begin{aligned}
& \frac{1}{2 \pi} \int_{-\alpha}^{\alpha} \frac{e^{\varepsilon a^{N}(u-i t)}}{u-i t} \varphi_{k}\left((u-i t) a^{N}\right) d t \\
& \quad \sim \Phi_{k, N} C_{k, N} \exp \left\{u \varepsilon a^{N}+v N \log S(\varphi(u))-\frac{1}{2} \log N\right\}
\end{aligned}
$$

as $\varepsilon \downarrow 0$, where

$$
\Phi_{k, N} \equiv \Phi_{k, N}(\varepsilon)=\frac{\varphi_{*}(u) F(\varphi(u))}{u \sqrt{2 \pi \nu \psi^{\prime \prime}(u)}} \Psi_{k, N}(\varphi(u)) .
$$

Proof. By our choice of $\beta$ we have $\varphi(u-i t) \in \mathcal{D}_{r, \theta}$ for all $t \in[-\beta, \beta]$. By Lemma 6.1 we obtain

$$
\varphi_{k}\left((u-i t) a^{N}\right) \sim \Psi_{k, N}(\varphi(u-i t)) \varphi_{*}(u-i t) F(\varphi(u-i t)) C_{k, N} S(\varphi(u-i t))^{v N}
$$


as $\varepsilon \downarrow 0$ uniformly for all $t \in[-\beta, \beta]$. Taking into account that $\alpha \downarrow 0$ we have

$$
\varphi_{k}\left((u-i t) a^{N}\right) \sim \Psi_{k, N}(\varphi(u)) \varphi_{*}(u) F(\varphi(u)) C_{k, N} S(\varphi(u-i t))^{\nu N}
$$

as $\varepsilon \downarrow 0$ for all $t \in[-\alpha, \alpha]$ since $\Psi_{k, N}$ is regular enough by Lemma 7.1. We obtain

$$
\begin{aligned}
& \int_{-\alpha}^{\alpha} \frac{e^{\varepsilon a^{N}(u-i t)}}{u-i t} \varphi_{k}\left((u-i t) a^{N}\right) d t \\
& \quad \sim C_{k, N} \Psi_{k, N}(\varphi(u)) \frac{\varphi_{*}(u) F(\varphi(u))}{u} \int_{-\alpha}^{\alpha} e^{\varepsilon a^{N}(u-i t)} S(\varphi(u-i t))^{\nu N} d t .
\end{aligned}
$$

For the integral above, we use the Taylor expansion (7.9) to obtain

$$
\begin{aligned}
& \int_{-\alpha}^{\alpha} e^{\varepsilon a^{N}(u-i t)} S(\varphi(u-i t))^{v N} d t \\
& \quad \sim \exp \left\{u \varepsilon a^{N}+v N \log S(\varphi(u))\right\} \int_{-\alpha}^{\alpha} \exp \left\{-i t\left(\varepsilon a^{N}+v N \psi^{\prime}(u)\right)-\frac{t^{2} v N \psi^{\prime \prime}(u)}{2}\right\} d t
\end{aligned}
$$

where we have also used $\alpha e^{N \alpha^{3}} \rightarrow 0$ from (7.11) to get rid of the negligible terms in the Taylor expansion. Observing that by (7.3) and (7.7)

$$
\begin{aligned}
\varepsilon a^{N}+v N \psi^{\prime}(u) & =\varepsilon a^{\rho-\{\rho\}}+v \rho \psi^{\prime}(u)-v\{\rho\} \psi^{\prime}(u) \\
& =\rho\left(a^{-\{\rho\}}+v \psi^{\prime}(u)\right)-v\{\rho\} \psi^{\prime}(u) \\
& =-v\{\rho\} \psi^{\prime}(u)
\end{aligned}
$$

we obtain

$$
\begin{aligned}
& \int_{-\alpha}^{\alpha} e^{\varepsilon a^{N}(u-i t)} S(\varphi(u-i t))^{\nu N} d t \\
& \quad \sim \exp \left\{u \varepsilon a^{N}+v N \log S(\varphi(u))\right\} \int_{-\alpha}^{\alpha} \exp \left\{i t v\{\rho\} \psi^{\prime}(u)-\frac{t^{2} v N \psi^{\prime \prime}(u)}{2}\right\} d t .
\end{aligned}
$$

Substituting $\tau=t \sqrt{v N \psi^{\prime \prime}(u)}$ we get

$$
\begin{aligned}
& \int_{-\alpha}^{\alpha} \exp \left\{i t v\{\rho\} \psi^{\prime}(u)-\frac{t^{2} v N \psi^{\prime \prime}(u)}{2}\right\} d t \\
& \sim \frac{1}{\sqrt{v N \psi^{\prime \prime}(u)}} \int_{-\alpha \sqrt{v N \psi^{\prime \prime}(u)}}^{\alpha \sqrt{v N \psi^{\prime \prime}(u)}} \exp \left\{\frac{i \tau \nu\{\rho\} \psi^{\prime}(u)}{\left.\sqrt{v N \psi^{\prime \prime}(u)}-\frac{\tau^{2}}{2}\right\} d \tau \sim \frac{\sqrt{2 \pi}}{\sqrt{v N \psi^{\prime \prime}(u)}}}\right.
\end{aligned}
$$


since, as $\varepsilon \downarrow 0$, the interval of integration increases to $\mathbb{R}$ by (7.11) and the first order term tends to zero (see for example [4], Lemma 11). Combining this with (7.14), we obtain

$$
\begin{aligned}
& \int_{-\alpha}^{\alpha} e^{\varepsilon a^{N}(u-i t)} S(\varphi(u-i t))^{v N} d t \\
& \sim \frac{\sqrt{2 \pi}}{\sqrt{\nu \psi^{\prime \prime}(u)}} \exp \left\{u \varepsilon a^{N}+v N \log S(\varphi(u))-\frac{1}{2} \log N\right\} .
\end{aligned}
$$

Together with (7.13), this proves the required asymptotics.

Lemma 7.3. Suppose that $k$ is of the form (7.1) or (7.2). Then

$$
\begin{aligned}
& \left|\int_{|t| \in[\alpha, \beta]} \frac{e^{\varepsilon a^{N}(u-i t)}}{u-i t} \varphi_{k}\left((u-i t) a^{N}\right) d t\right| \\
& \quad \leq C_{k, N} \exp \left\{u \varepsilon a^{N}+v N \log S(\varphi(u))-\log N\right\},
\end{aligned}
$$

for all \& small enough.

Proof. Observe that (7.12) is in particular true for all $t$ such that $|t| \in[\alpha, \beta]$. Since $|u-i t| \geq u_{*}$, $\left|\varphi_{*}(u-i t)\right| \leq 1, F$ is bounded by Lemma 4.2 , and $\Psi_{k, N}$ is uniformly bounded by Lemma 7.1, there is a positive constant $c$ such that

$$
\begin{aligned}
& \left|\int_{|t| \in[\alpha, \beta]} \frac{e^{\varepsilon a^{N}(u-i t)}}{u-i t} \varphi_{k}\left((u-i t) a^{N}\right) d t\right| \\
& \quad \leq c C_{k, N}\left|\int_{|t| \in[\alpha, \beta]} e^{\varepsilon a^{N}(u-i t)} S(\varphi(u-i t))^{v N} d t\right| .
\end{aligned}
$$

For the integral above, we use the Taylor bound (7.10) to obtain

$$
\begin{aligned}
& \left|\int_{|t| \in[\alpha, \beta]} e^{\varepsilon a^{N}(u-i t)} S(\varphi(u-i t))^{v N} d t\right| \\
& \quad \leq \exp \left\{u \varepsilon a^{N}+v N \log S(\varphi(u))\right\} \int_{|t| \in[\alpha, \beta]} \exp \left\{-\frac{t^{2} v N \psi^{\prime \prime}(u)}{4}\right\} d t \\
& \quad \leq 2 \beta \exp \left\{u \varepsilon a^{N}+v N \log S(\varphi(u))-\frac{\alpha^{2} v N \psi^{\prime \prime}(u)}{4}\right\} .
\end{aligned}
$$

Taking into account the fact that $\alpha^{2} N / \log N \rightarrow \infty$ according to (7.11) and combining (7.15) with (7.16), we obtain the desired estimate. 
Lemma 7.4. Suppose that $k$ is of the form (7.1) or (7.2). There exists $\delta>0$ such that

$$
\begin{aligned}
& \left|\int_{|t| \geq \beta} \frac{e^{\varepsilon a^{N}(u-i t)}}{u-i t} \varphi_{k}\left((u-i t) a^{N}\right) d t\right| \\
& \quad \leq C_{k, N} \exp \left\{u \varepsilon a^{N}+v N \log S(\varphi(u))-\delta v N\right\}
\end{aligned}
$$

and

$$
\begin{aligned}
& \left|\int_{-\infty}^{\infty} \frac{1}{u-i t} \varphi_{k}\left((u-i t) a^{N}\right) d t\right| \\
& \quad \leq C_{k, N} \exp \{v N \log S(\varphi(u))-\delta v N\}
\end{aligned}
$$

for all \& small enough.

Proof. Similarly to the proof of [10], Lemma 16, we use the fact that, for each $v \in\left[u_{*}, u^{*}\right]$, $t \mapsto \varphi(v-i t) / \varphi(v)$ is the characteristic function of some absolutely continuous law (Cramér transform), the continuity of the mapping $(v, t) \mapsto \varphi(v-i t) / \varphi(v)$, and the compactness of $\left[u_{*}, u^{*}\right]$ to conclude that there is a constant $\eta$ such that

$$
|\varphi(u-i t)| \leq(1-\eta) \varphi(u) \quad \text { for all }|t| \geq \beta
$$

and all $\varepsilon$ small enough. Using Lemma 6.1 with $s \equiv s(\varepsilon)=(1-\eta) \varphi(u)<\varphi\left(u_{*}\right)<r$ and taking into account that $s \geq(1-\eta) \varphi\left(u^{*}\right)$ and so is separated from zero, we obtain, with some positive constant $c_{1}$,

$$
\left|\varphi_{k}\left((u-i t) a^{N}\right)\right| \leq c_{1}|\varphi(u-i t)| C_{k, N} S((1-\eta) \varphi(u))^{v N} .
$$

By Lemma 5.1 and the mean value theorem

$$
\log S((1-\eta) \varphi(u)) \leq \log S(\varphi(u))-\eta,
$$

which implies

$$
\left|\varphi_{k}\left((u-i t) a^{N}\right)\right| \leq c_{1}|\varphi(u-i t)| C_{k, N} S(\varphi(u))^{v N} e^{-\eta v N} .
$$

Substituting this estimate into the integral (7.17) and using $|u-i t| \geq u_{*}$ we obtain

$$
\begin{aligned}
& \left|\int_{|t| \geq \beta} \frac{e^{\varepsilon a^{N}(u-i t)}}{u-i t} \varphi_{k}\left((u-i t) a^{N}\right) d t\right| \\
& \quad \leq c_{2} C_{k, N} \exp \left\{u \varepsilon a^{N}+v N \log S(\varphi(u))-\eta v N\right\} \int_{-\infty}^{\infty}|\varphi(u-i t)| d t
\end{aligned}
$$

with some $c_{2}>0$. It was shown in [10], Lemma 16, that the integral above is uniformly bounded. This implies the required estimate with some $\delta<\eta$.

The estimate (7.18) is obtained in the same way as (7.17) with the only difference that the terms $e^{\varepsilon a^{N}(u-i t)}$ and $u \varepsilon a^{N}$ are omitted. 


\section{Proofs of the main theorems}

In this section, we establish the joint probability (6.7) and then prove Theorems 1.2 and 1.3.

Proposition 8.1. Suppose that $k$ is of the form (7.1) or (7.2). As $\varepsilon \downarrow 0$,

$$
\mathbb{P}\{\mathcal{K}>k, \mathcal{W}<\varepsilon\} \sim q_{\nu}^{N} p_{1}^{\frac{v N(N+1)}{2}} \Phi_{k, N} \exp \left\{u \varepsilon a^{N}+v N \log S(\varphi(u))-\frac{1}{2} \log N\right\} .
$$

Proof. Combining (6.7) and (7.8) we obtain

$$
\mathbb{P}\{\mathcal{K}>k, \mathcal{W}<\varepsilon\}=q_{v}^{k+1} p_{1}^{\frac{\nu k(k+1)}{2}} \frac{1}{2 \pi} \int_{-\infty}^{\infty} \frac{e^{\varepsilon a^{N}(u-i t)}-1}{u-i t} \varphi_{k}\left((u-i t) a^{N}\right) d t .
$$

Let us split the integral into the sum of the three integrals corresponding to keeping $e^{\varepsilon a^{N}(u-i t)}$ in the numerator and integrating over $[-\alpha, \alpha],\{t:|t| \in[\alpha, \beta]\}$, and $\{t:|t| \geq \beta\}$, respectively, and the integral corresponding to keeping -1 in the numerator and integrating over $\mathbb{R}$. Lemma 7.2 gives the asymptotics of the first integral, while Lemmas 7.3 and 7.4 imply that the remaining three integrals are negligible since $\Psi_{k, N}$ is bounded away from zero and infinity by Lemma 7.1. Substituting the asymptotics for the first integral given by Lemma 7.2 we arrive at the required formula.

Proof of Theorem 1.2. We use Proposition 8.1 with $k=-1$ as

$$
\mathbb{P}\{\mathcal{W}<\varepsilon\}=\mathbb{P}\{\mathcal{K}>-1, \mathcal{W}<\varepsilon\} .
$$

By Lemma 7.1, we have

$$
\Phi_{-1, N} \sim \frac{\varphi_{*}(u) F(\varphi(u))}{u \sqrt{2 \pi \nu \psi^{\prime \prime}(u)}}
$$

Using (7.3), we get

$$
\begin{aligned}
u \varepsilon a^{N} & =u \varepsilon a^{\rho-\{\rho\}}=u \rho a^{-\{\rho\}}, \\
v N \log S(\varphi(u)) & =v \rho \log S(\varphi(u))-v\{\rho\} \log S(\varphi(u)), \\
\log N & =\log \rho+o(1) .
\end{aligned}
$$

Further,

$$
\begin{aligned}
q_{v}^{N} & =\exp \left\{\rho \log q_{v}-\{\rho\} \log q_{\nu}\right\}, \\
p_{1}^{\frac{v N(N+1)}{2}} & =\exp \left\{\frac{\nu \log p_{1}}{2}\left(\rho^{2}+\rho(1-2\{\rho\})+\{\rho\}^{2}-\{\rho\}\right)\right\} .
\end{aligned}
$$


Substituting all of the above into (8.1), we obtain the required asymptotics (1.6) with

$$
\begin{aligned}
& M_{1}(\omega)=\frac{1}{\log a} \hat{M}_{1}\left(\frac{\omega}{\log a}\right), \\
& M_{2}(\omega)=\frac{1}{2} \log \log a+\hat{M}_{2}\left(\frac{\omega}{\log a}\right),
\end{aligned}
$$

where

$$
\begin{aligned}
& \hat{M}_{1}(\rho)=\frac{v \log p_{1}}{2}(1-2\{\rho\})+u a^{-\{\rho\}}+v \log S(\varphi(u))+\log q_{\nu}, \\
& \hat{M}_{2}(\rho)=\log \left[\frac{\varphi_{*}(u) F(\varphi(u))}{u \sqrt{2 \pi \nu \psi^{\prime \prime}(u)}}\right]+\frac{\nu \log p_{1}}{2}\left(\{\rho\}^{2}-\{\rho\}\right)-v\{\rho\} \log S(\varphi(u))-\{\rho\} \log q_{\nu},
\end{aligned}
$$

which are bounded periodic functions of $\rho$ with period one since $u$ is a bounded function of $\{\rho\}$ by (7.7).

Proof of Theorem 1.3. Let $x \in \mathbb{Z}$. Using Proposition 8.1 both with $k=-1$ and $k \equiv k(\varepsilon)=$ $\lfloor\gamma\rfloor+x$ we obtain

$$
\begin{aligned}
\mathbb{P}\{\mathcal{K}>\lfloor\gamma\rfloor+x \mid \mathcal{W}<\varepsilon\} & =\frac{\mathbb{P}\{\mathcal{K}>\lfloor\gamma\rfloor+x, \mathcal{W}<\varepsilon\}}{\mathbb{P}\{\mathcal{K}>-1, \mathcal{W}<\varepsilon\}} \sim \frac{\Phi_{\lfloor\gamma\rfloor+x, N}}{\Phi_{-1, N}} \\
& =\frac{\Psi_{\lfloor\gamma\rfloor+x, N}(\varphi(u))}{\Psi_{-1, N}(\varphi(u))} \sim \exp \left\{-w \nu p_{1}^{-(\lambda-1) x} S(\varphi(u))^{\lambda-1}\right\}
\end{aligned}
$$

by Lemma 7.1. This implies (1.8) and (1.9) with

$$
c_{1}=v \liminf _{\varepsilon \downarrow 0}\left[w S(\varphi(u))^{\lambda-1}\right] \quad \text { and } \quad c_{2}=v \limsup _{\varepsilon \downarrow 0}\left[w S(\varphi(u))^{\lambda-1}\right]
$$

which are both positive and finite since $S \circ \varphi$ is continuous on $\left[u_{*}, u^{*}\right]$ and $w$ is bounded away from zero and infinity by Lemma 7.1.

\section{Acknowledgement}

The author was supported by the Leverhulme Research Grant RPG-2012-608.

\section{References}

[1] Asmussen, S. and Hering, H. (1983). Branching Processes. Progress in Probability and Statistics 3. Boston, MA: Birkhäuser, Inc. MR0701538

[2] Athreya, K.B. and Ney, P.E. (1970). Branching Processes. Berlin: Springer.

[3] Barlow, M.T. and Perkins, E.A. (1988). Brownian motion on the Sierpiński gasket. Probab. Theory Related Fields 79 543-623. MR0966175 
[4] Berestycki, N., Gantert, N., Mörters, P. and Sidorova, N. (2014). Galton-Watson trees with vanishing martingale limit. J. Stat. Phys. 155 737-762. MR3192182

[5] Biggins, J.D. and Bingham, N.H. (1993). Large deviations in the supercritical branching process. Adv. Appl. Probab. 25 757-772.

[6] Chu, W. (2014). Small value probabilities for supercritical multitype branching processes with immigration. Statist. Probab. Lett. 93 87-95.

[7] Chu, W., Li, W. and Ren, Y.-X. (2014). Small value probabilities for supercritical branching processes with immigration. Bernoulli 20 377-393.

[8] Dubuc, S. (1971). Problémes relatifs á l'itération de fonctions suggérés par les processus en cascade. Ann. Inst. Fourier (Grenoble) 21 171-251.

[9] Dubuc, S. (1971). La densité de la loi limite d'un processus en cascade expansif. Z. Wahrscheinlichkeitsth. 19 281-290.

[10] Fleischmann, K. and Wachtel, V. (2009). On the left tail asymptotics for the limit law of supercritical Galton-Watson processes in the Böttcher case. Ann. Inst. Henri Poincaré Probab. Stat. 45 201-225.

[11] Hambly, B.M. (1995). On constant tail behaviour for the limiting random variable in a supercritical branching process. J. Appl. Probab. 32 267-273. MR1316808

[12] Lifshits, M. (2006). Bibliography of small deviation probabilities. Updated version downloadable from http://www.proba.jussieu.fr/pageperso/smalldev/biblio.pdf.

[13] Seneta, E. (1970). On the supercritical Galton-Watson process with immigration. Math. Biosci. 7 $9-14$.

Received December 2016 and revised June 2017 